\title{
WELL-POSEDNESS FOR A CLASS OF NON-NEWTONIAN FLUIDS WITH GENERAL GROWTH CONDITIONS
}

\author{
PIOTR GWIAZDA, AGNIESZKA ŚWIERCZEWSKA-GWIAZDA, ANETA WRÓBLEWSKA \\ Institute of Applied Mathematics and Mechanics, Warsaw University \\ Banacha 2, 02-097 Warszawa, Poland \\ E-mail: pgwiazda@mimuw.edu.pl,aswiercz@mimuw.edu.pl, \\ A.Wroblewska@students.mimuw.edu.pl
}

ANDRZEJ WARZYŃSKI

Faculty of Mathematics and Information Science Warsaw University of Technology

Pl. Politechniki 1, 00-661 Warszawa, Poland

E-mail: A.Warzynski@students.mimuw.edu.pl

\begin{abstract}
The paper concerns uniqueness of weak solutions to non-Newtonian fluids with nonstandard growth conditions for the Cauchy stress tensor. We recall the results on existence of weak solutions and additionally provide the proof of existence of measure-valued solutions. Motivated by the fluids of strongly inhomogeneous behaviour and having the property of rapid shear thickening we observe that the described situation cannot be captured by power-lawtype rheology. We describe the growth conditions with the help of general $x$-dependent convex functions. This formulation yields the existence of solutions in generalized Orlicz spaces. These considerations are motivated by e.g. electrorheological fluids, magnetorheological fluids, and shear thickening fluids.
\end{abstract}

1. Introduction. We investigate the behavior of non-Newtonian fluids and rapidly shear thickening fluids. Our interest is directed to the phenomena of viscosity increase under various stimuli: shear rate, magnetic or electric field. All those fluids are of colloid type, hence e.g. under applying the magnetic field, the iron particles suspended in the liquid form column-like structures parallel to the magnetic field. Both magnetorheological and shear thickening fluids have an ability of transferring rapidly from liquid to solid

2000 Mathematics Subject Classification: 35K55, 35Q35, 46E30.

Key words and phrases: non-Newtonian fluids, Orlicz spaces, modular convergence, smart fluids, measure-valued solutions, weak solutions, uniqueness.

The paper is in final form and no version of it will be published elsewhere. 
state. We will briefly mention some applications of such fluids in industry, military and natural science. The magnetorheological fluids are used for modern suspension systems, clutches or crash-protection systems in cars. Moreover they are used in shock absorbers providing seismic protection. Another possible application, however still on the level of laboratory research, appears in military armor. The so-called liquid body armor consists in soaking the armor material with fluid which increases the viscosity under the stimulus within few milliseconds. This allows for improving the flexibility and reducing the weight of the protection. For the liquid body armor, more promising solution for the moment are shear thickening fluids. For more detailed description of the model, examples and references see [4].

The fluids in question are described by the equations

$$
\begin{aligned}
v_{t}+(v \cdot \nabla) v-\operatorname{div} S(x, D v)+\nabla \pi & =f \quad \text { in } \quad Q, \\
\operatorname{div} v & =0 \quad \text { in } \quad Q, \\
v(0, x) & =v_{0} \text { in } \Omega, \\
v(t, x) & =0 \quad \text { on } \quad(0, T) \times \partial \Omega,
\end{aligned}
$$

where $v: Q \rightarrow \mathbb{R}^{d}$ denotes the velocity field, $\pi: Q \rightarrow \mathbb{R}$ the pressure, $S$ the stress tensor, $f: Q \rightarrow \mathbb{R}^{d}$ the body forces, $\Omega \subset \mathbb{R}^{d}$ is a bounded domain with a smooth boundary $\partial \Omega$ and we denote by $Q=(0, T) \times \Omega$ with some given $T>0$ and $D v=\frac{1}{2}\left(\nabla v+\nabla^{T} v\right)$.

The anisotropic character of the flow and rapid viscosity growth do not fall under the polynomial description of the flow, i.e.

$$
\begin{aligned}
& |S(x, \xi)| \leq c(1+|\xi|)^{p-1}, \\
& S(x, \xi) \cdot \xi \geq c|\xi|^{p} .
\end{aligned}
$$

We formulate non-standard growth conditions with the help of more general convex functions. Before we formulate the assumptions on $S$ let us introduce some notions (adjusted to further applications). A function $M: \Omega \times \mathbb{R}_{\mathrm{sym}}^{d \times d} \rightarrow \mathbb{R}_{+}$is called an $N$-function if it is a convex (w.r.t. $\xi$ ) Carathéodory function such that $M(x, \xi)=0$ if and only if $\xi=0$, and $M(x, \xi)=M(x,-\xi)$ a.e. in $\Omega$. Moreover

$$
\lim _{|\xi| \rightarrow 0} \sup _{x \in \Omega} \frac{M(x, \xi)}{|\xi|}=0 \text { and } \quad \lim _{|\xi| \rightarrow \infty} \inf _{x \in \Omega} \frac{M(x, \xi)}{|\xi|}=\infty .
$$

The complementary function $M^{*}$ to a function $M$ is defined by

$$
M^{*}(x, \eta)=\sup _{\xi \in \mathbb{R}_{\mathrm{sym}}^{d \times d}}(\xi \cdot \eta-M(x, \xi))
$$

for $\eta \in \mathbb{R}_{\mathrm{sym}}^{d \times d}, x \in \Omega$. The complementary function $M^{*}$ is again an $N$-function.

We suppose that $S: \Omega \times \mathbb{R}_{\mathrm{sym}}^{d \times d} \rightarrow \mathbb{R}_{\mathrm{sym}}^{d \times d}$ satisfies $\left(\mathbb{R}_{\mathrm{sym}}^{d \times d}\right.$ stands for the space of $d \times d$ symmetric matrices):

(S1) $S(x, \xi)$ is a Carathéodory function (i.e., measurable function of $x$ for all $\xi \in \mathbb{R}_{\mathrm{sym}}^{d \times d}$ and continuous function of $\xi$ for a.a. $x \in \Omega)$ and $S(x, 0)=0$.

(S2) There exist a positive constant $c, N$-functions $M$ and $\tilde{M}$ such that for all $\xi \in \mathbb{R}_{\mathrm{sym}}^{d \times d}$ and a.a. $x \in \Omega$,

$$
S(x, \xi) \cdot \xi \geq c\{M(x, \xi)+\tilde{M}(x, S(x, \xi))\}
$$


(S3) There exists $c>0$ and $p>1$ such that

$$
M(x, \xi) \geq c|\xi|^{p} .
$$

(S4) $S$ is monotone, i.e. for all $\xi_{1}, \xi_{2} \in \mathbb{R}_{\mathrm{sym}}^{d \times d}$, and a.a. $x \in \Omega$

$$
\left[S\left(x, \xi_{1}\right)-S\left(x, \xi_{2}\right)\right] \cdot\left[\xi_{1}-\xi_{2}\right] \geq 0 .
$$

The power-law-type growth conditions (1.2) impose the formulation of the problem in $L^{p}$ spaces. To consider the problem (1.1) with assumptions (S1)-(S4) on the stress tensor $S$ we will use the $N$-function $M$ to define generalized Orlicz spaces. Let us first define the generalized Orlicz class $\mathcal{L}_{M}(Q)$ as the set of all measurable functions $\xi: Q \rightarrow \mathbb{R}_{\mathrm{sym}}^{d \times d}$ such that

$$
\int_{Q} M(x, \xi) d x d t<\infty .
$$

By $L_{M}(Q)$ we denote the generalized Orlicz space which is the set of all measurable functions $\xi: Q \rightarrow \mathbb{R}_{\mathrm{sym}}^{d \times d}$ which satisfy

$$
\int_{Q} M(x, \lambda \xi(x)) d x d t \rightarrow 0 \quad \text { as } \lambda \rightarrow 0 .
$$

The generalized Orlicz space is a Banach space with respect to the Orlicz norm

$$
\|\xi\|_{O}=\sup \left\{\int_{Q} \eta \cdot \xi d x d t: \eta \in L_{M^{*}}(Q), \int_{Q} M^{*}(x, \eta) d x d t \leq 1\right\}
$$

or the equivalent Luxemburg norm

$$
\|\xi\|_{L}=\inf \left\{\lambda>0: \quad \int_{Q} M\left(x, \frac{\xi}{\lambda}\right) d x d t \leq 1\right\} .
$$

The functional

$$
\rho(\xi)=\int_{Q} M(x, \xi(x)) d x d t
$$

is a modular, see e.g. [10] for definition. We will say that a sequence $z^{j}$ converges modularly to $z$ in $L_{M}(Q)$ if there exists $\lambda>0$ such that

$$
\int_{Q} M\left(x, \frac{z^{j}-z}{\lambda}\right) d x d t \rightarrow 0 .
$$

We will use the notation $z^{j} \stackrel{M}{\longrightarrow} z$ for the modular convergence in $L_{M}(Q)$. We call these function spaces generalized Orlicz spaces since in contrast to classical Orlicz spaces we allow the function $M$ to depend on $x$ and the whole vector $\xi$, not only on $|\xi|$. The generalized Orlicz class is equal to the generalized Orlicz space if the $N$-function satisfies the $\Delta_{2}$-condition, i.e. for some nonnegative function $h$ integrable in $\Omega$ and a constant $k>0$

$$
M(x, 2 \xi) \leq k M(x, \xi)+h(x) \quad \text { for all } \xi \in \mathbb{R}_{\text {sym }}^{d \times d} \text { and a.a. } x \in \Omega .
$$

Since we are interested in stresses of growth faster than polynomial, the $N$-function describing the growth of $S$ does not satisfy the $\Delta_{2}$-condition. This results in additional analytical difficulties like the lack of separability, reflexivity and density of smooth functions in the space $L_{M}(Q)$. 
The paper is organized as follows: Section 2 is devoted to measure-valued solutions (Theorem 2.1); in Section 3 we briefly recall the results on existence and continuity of weak solutions in Theorem 3.2. The last section concerns the uniqueness of weak solutions (Theorem 4.1) which is the main result of the present paper and settles the problem of well posedness of the solution to (1.1).

The uniqueness of the flow for power-law-type fluids was extensively studied together with the question of existence of solutions. The first results were provided by Ladyzhenskaya and Lions, cf. $[6,7]$ for $p \geq(d+2) / 2$ and $v_{0} \in L_{\text {div }}^{2}(\Omega)$ and later extended to $p \geq 1+2 d /(d+2)$ and $v_{0} \in W_{0, \text { div }}^{1,2}(\Omega)$, see [8]. Recent studies relax the assumptions on $p$ and provide the uniqueness unless $p>(3 d+2) / d+2$, cf. [2]. The latter paper includes the dependence of the stress tensor on $x$. In our analysis we need to overcome the problem that the solution is not an admissible test function, which results in technical difficulties.

Finally notice that if we neglect the convective term in the equations, then condition (S3) could be relaxed. The presented analysis however needs an essential modification if we want to include the models of very slow rate characterizing the dependence of the viscosity on the shear rate. This relates to the fact that (S3) implies that $M^{*}$ satisfies the $\Delta_{2}$-condition.

By $\mathcal{D}(\Omega)$ we denote the set of $C^{\infty}$-functions with compact support. Let $\mathcal{V}(\Omega)$ be the set of all functions which belong to $\mathcal{D}(\Omega)$ and are divergence-free. Moreover by $L^{q}, W^{1, q}$ we denote the standard Lebesgue and Sobolev spaces, by $L_{\text {div }}^{2}$ the closure of $\mathcal{V}$ w.r.t. the $\|\cdot\|_{2}$ norm and by $W_{0, \text { div }}^{1, p}$ the closure of $\mathcal{V}$ w.r.t. the $\|\nabla(\cdot)\|_{p}$ norm. By $p^{\prime}$ we denote the conjugate exponent to $p$, namely $\frac{1}{p}+\frac{1}{p^{\prime}}=1$.

2. Measure-valued solutions. In this section we establish the existence of a measurevalued solution to the time-dependent model (1.1). This naturally requires weaker assumptions than the proof of the existence of weak solutions (see Section 3). Here, we can consider $p>\frac{2 d}{d+2}$ and we do not assume that $S$ is monotone.

THEOREM 2.1. Let $S$ satisfy conditions (S1)-(S3) with $p>\frac{2 d}{d+2}$ and let $v_{0} \in L_{\text {div }}^{2}(\Omega), f \in$ $L^{p^{\prime}}\left(I,\left(W_{0}^{1, p}(\Omega)\right)^{*}\right), f=\operatorname{div} F$ with $F \in L_{M^{*}}(Q)$. Then there exists a measure-valued solution $(v, \nu)$, i.e.,

$$
\begin{gathered}
v \in L^{\infty}\left(0, T ; L^{2}(\Omega)\right) \cap L^{p}\left(0, T ; W_{0, \operatorname{div}}^{1, p}(\Omega)\right), \\
\nu \in L^{\infty}\left(Q ; \mathcal{M}\left(\mathbb{R}^{d \times d}\right)\right),
\end{gathered}
$$

and the identity

$$
\begin{aligned}
& \int_{0}^{T}\left[\int_{\Omega}\left(v \cdot \varphi_{t}+v \otimes v \cdot \nabla \varphi\right) d x+\langle f, \varphi\rangle\right] d t+\int_{\Omega} v_{0} \cdot \varphi(0) d x \\
& =\int_{Q} \int_{\mathbb{R}^{d \times d}} S\left(x, \frac{\xi+\xi^{T}}{2}\right) d \nu_{t, x}(\xi) \cdot D \varphi d x d t
\end{aligned}
$$

is satisfied for all $\varphi \in \mathcal{D}((-\infty, T) ; \mathcal{V})$. Moreover

$$
\nabla v(t, x)=\int_{\mathbb{R}^{d \times d}} \xi d \nu_{t, x}(\xi) \quad \text { for a.a. } \quad(t, x) \in Q
$$


and for almost all $\tau \in[0, T)$

$$
\begin{aligned}
& \frac{1}{2}\|v(\tau)\|_{2}^{2}+\int_{Q_{\tau}} \int_{\mathbb{R}^{d \times d}} S\left(x, \frac{\xi+\xi^{T}}{2}\right) \cdot\left(\frac{\xi+\xi^{T}}{2}\right) d \nu_{t, x}(\xi) d x d t \\
& \leq \frac{1}{2}\left\|v_{0}\right\|_{2}^{2}+\int_{0}^{\tau}\langle f, v\rangle d t, \quad Q_{\tau}:=(0, \tau) \times \Omega .
\end{aligned}
$$

Proof. The first part of the proof follows the same lines as [4]. However we recall it for completeness.

We construct Galerkin approximations to (1.1). First, we describe the chosen basis $\left\{\omega_{i}\right\}$. Assume that $s>\frac{d}{2}+1$ and denote

$$
V_{s} \equiv \text { the closure of } \mathcal{V} \text { w.r.t. the } W^{s, 2}(\Omega) \text {-norm. }
$$

Let then the scalar product in $V_{s}$ be denoted by $((\cdot, \cdot))_{s}$ and $\left\{\omega_{i}\right\}$ be the set of eigenvectors to the problem

$$
\left(\left(\omega_{i}, \varphi\right)\right)_{s}=\lambda_{i}\left(\omega_{i}, \varphi\right) \text { for all } \varphi \in V_{s} .
$$

Notice that for $s$ specified above the Sobolev embedding theorem provides

$$
W^{s-1,2}(\Omega) \hookrightarrow L^{\infty}(\Omega)
$$

which we will use in the sequel. We define $v^{n}=\sum_{i=1}^{n} \alpha_{i}^{n}(t) \omega_{i}$, where $\alpha_{i}^{n}(t)$ solve the system

$$
\begin{aligned}
\int_{\Omega} \frac{d}{d t} v^{n} \cdot \omega_{i} d x+\int_{\Omega}\left(v^{n} \cdot \nabla\right) v^{n} \cdot \omega_{i} d x+\int_{\Omega} S\left(x, D v^{n}\right) \cdot D \omega_{i} d x & =\left\langle f, \omega_{i}\right\rangle, \\
v^{n}(0) & =P^{n} v_{0},
\end{aligned}
$$

$1 \leq i \leq n$. By $P^{n}$ we denote the orthogonal projection of $L_{\mathrm{div}}^{2}(\Omega)$ on $\operatorname{conv}\left\{\omega_{1}, \ldots, \omega_{n}\right\}$. Multiplying each equation of (2.13) by $\alpha_{i}^{n}(t)$, summing over $i=1, \ldots, n$ and remembering that since $\operatorname{div} v^{n}=0$, then $\int_{\Omega}\left(v^{n} \cdot \nabla\right) v^{n} \cdot v^{n} d x=0$, we obtain

$$
\frac{1}{2} \frac{d}{d t}\left\|v^{n}\right\|_{2}^{2}+\int_{\Omega} S\left(x, D v^{n}\right) \cdot D v^{n} d x=\left\langle f, v^{n}\right\rangle .
$$

If (1.5) holds, then one easily shows there exists some $c>0$ such that

$$
M^{*}(x, \xi) \leq c|\xi|^{p^{\prime}},
$$

which implies that $M^{*}$ satisfies the $\Delta_{2}$-condition. Consequently, by Proposition A.5, we conclude that $F \in \mathcal{L}_{M^{*}}(Q)$ and estimate

$$
\begin{aligned}
\left|\left\langle\operatorname{div} F, v^{n}\right\rangle\right| & \leq \int_{\Omega}\left|\frac{2}{c} F \cdot \frac{c}{2} D v^{n}\right| d x \\
& \stackrel{F-Y}{\leq} \int_{\Omega} M^{*}\left(x, \frac{2}{c} F\right) d x+\int_{\Omega} M\left(x, \frac{c}{2} D v^{n}\right) d x \\
& \leq \int_{\Omega} M^{*}\left(x, \frac{2}{c} F\right) d x+\frac{c}{2} \int_{\Omega} M\left(x, D v^{n}\right) d x
\end{aligned}
$$

Integrating (2.14) over the time interval $(0, \tau)$, using estimate (2.16) and the coercivity 
conditions (1.4) on $S$ we obtain

$$
\begin{aligned}
\frac{1}{2}\left\|v^{n}(\tau)\right\|_{2}^{2} & +\frac{c}{2} \int_{0}^{\tau} \int_{\Omega} M\left(x, D v^{n}\right) d x d t+c \int_{0}^{\tau} \int_{\Omega} \tilde{M}\left(x, S\left(x, D v^{n}\right)\right) d x d t \\
& \leq \int_{0}^{\tau} \int_{\Omega} M^{*}\left(x, \frac{2}{c} F\right) d x d t+\frac{1}{2}\left\|v_{0}^{n}\right\|_{2}^{2},
\end{aligned}
$$

for all $\tau \in(0, T]$. The condition (1.5) implies that $\left\{D v^{n}\right\}$ is uniformly bounded in the space $L^{p}(Q)$ for $p>\frac{2 d}{d+2}$ and hence there exists a subsequence such that

$$
D v^{n} \rightarrow D v \quad \text { weakly in } L^{p}(Q) .
$$

Using (2.17) we get that the sequence $\left\{S\left(\cdot, D v^{n}\right)\right\}$ is uniformly bounded in the Orlicz class $\mathcal{L}_{\tilde{M}}(Q)$. Applying Lemma A.7 we deduce the uniform integrability and hence weak precompactness in $L^{1}(Q)$. Namely there exists a $\chi \in L^{1}(Q)$ such that

$$
S\left(\cdot, D v^{n}\right) \rightarrow \chi \quad \text { weakly in } \quad L^{1}(Q) .
$$

Moreover from (2.17) we deduce uniform boundedness of the sequence $v^{n}$ in the space $L^{\infty}\left(0, T ; L_{\text {div }}^{2}(\Omega)\right)$ and as an immediate conclusion, we have

$$
v^{n} \stackrel{*}{\rightarrow} v \quad \text { in } L^{\infty}\left(0, T ; L_{\mathrm{div}}^{2}(\Omega)\right) .
$$

To establish the uniform bound for $\frac{d v^{n}}{d t}$ we take a test function $\varphi \in L^{\infty}\left(0, T ; V_{s}\right)$. Observe that

$$
\begin{aligned}
& \left\langle\frac{d v^{n}}{d t}, \varphi\right\rangle=\left\langle\frac{d v^{n}}{d t}, P^{n} \varphi\right\rangle \\
& \quad=-\int_{\Omega} S\left(x, D v^{n}\right) \cdot D\left(P^{n} \varphi\right) d x-\int_{\Omega}\left(v^{n} \cdot \nabla\right) v^{n} \cdot P^{n} \varphi d x+\left\langle\operatorname{div} F, P^{n} \varphi\right\rangle .
\end{aligned}
$$

Using that $\left\|P^{n} \varphi\right\|_{V_{s}} \leq\|\varphi\|_{V_{s}}$ and (2.12) we estimate the following integrals:

$$
\begin{gathered}
\left|\int_{0}^{T} \int_{\Omega} S\left(x, D v^{n}\right) \cdot D\left(P^{n} \varphi\right) d x d t\right| \leq \int_{0}^{T}\left\|S\left(\cdot, D v^{n}\right)\right\|_{1}\left\|D\left(P^{n} \varphi\right)\right\|_{\infty} d t \\
\leq \int_{0}^{T}\left\|S\left(\cdot, D v^{n}\right)\right\|_{1}\left\|P^{n} \varphi\right\|_{V_{s}} d t \leq \int_{0}^{T}\left\|S\left(\cdot, D v^{n}\right)\right\|_{1}\|\varphi\|_{V_{s}} d t \\
\leq\left\|S\left(\cdot, D v^{n}\right)\right\|_{L^{1}(Q)}\|\varphi\|_{L^{\infty}\left(0, T ; V_{s}\right)}
\end{gathered}
$$

and

$$
\begin{aligned}
\mid \int_{0}^{T} \int_{\Omega} & \left(v^{n} \cdot \nabla\right) v^{n} \cdot P^{n} \varphi d x d t|=| \int_{0}^{T} \int_{\Omega}\left(v^{n} \otimes v^{n}\right) \cdot \nabla P^{n} \varphi d x d t \mid \\
& \leq \int_{0}^{T}\left\|v^{n} \otimes v^{n}\right\|_{1}\left\|\nabla P^{n} \varphi\right\|_{\infty} d t \\
& \leq \int_{0}^{T}\left\|v^{n}\right\|_{2}^{2}\left\|P^{n} \varphi\right\|_{V_{s}} d t \leq \int_{0}^{T}\left\|v^{n}\right\|_{2}^{2}\|\varphi\|_{V_{s}} d t \\
& \leq\left\|v^{n}\right\|_{L^{2}(Q)}^{2}\|\varphi\|_{L^{\infty}\left(0, T ; V_{s}\right)} .
\end{aligned}
$$


To handle the right-hand side term, recall that $f=\operatorname{div} F$. Thus

$$
\begin{gathered}
\left|\int_{0}^{T}\left\langle\operatorname{div} F, P^{n} \varphi\right\rangle d t\right|=\left|\int_{0}^{T} \int_{\Omega} F \cdot D\left(P^{n} \varphi\right) d x d t\right| \\
\quad \leq \int_{0}^{T}\|F\|_{1}\left\|D\left(P^{n} \varphi\right)\right\|_{\infty} d t \leq \int_{0}^{T}\|F\|_{1}\left\|P^{n} \varphi\right\|_{V_{s}} d t \\
\leq \int_{0}^{T}\|F\|_{1}\|\varphi\|_{V_{s}} d t \leq\|F\|_{L^{1}(Q)}\|\varphi\|_{L^{\infty}\left(0, T ; V_{s}\right)} .
\end{gathered}
$$

Hence we conclude that $\frac{d v^{n}}{d t}$ is bounded in $L^{1}\left(0, T ; V_{s}^{*}\right)$. Because of the low regularity of the time derivative we recall the following generalization of the classical Aubin-Lions lemma, cf. [12]. We use the notation

$$
W^{1, p, q}\left(I ; X_{1}, X_{2}\right):=\left\{u \in L^{p}\left(I ; X_{1}\right) ; \frac{d u}{d t} \in L^{q}\left(I ; X_{2}\right)\right\}
$$

for $X_{1}$ a Banach space and $X_{2}$ a locally convex space, $X_{1} \subset X_{2}$. By $\frac{d u}{d t}$ we denote the distributional derivative, $\hookrightarrow$ means a continuous embedding and $\hookrightarrow \hookrightarrow$ a compact embedding.

Lemma 2.2 (Aubin-Lions, generalized). Let $X_{1}, X_{2}, X_{3}$ be Banach spaces, $X_{1}$ be separable and reflexive, $X_{1} \hookrightarrow \hookrightarrow X_{2}, X_{2} \hookrightarrow X_{3}, 1<p<\infty, 1 \leq q \leq \infty$. Then

$$
W^{1, p, q}\left(I ; X_{1}, X_{3}\right) \hookrightarrow \hookrightarrow L^{p}\left(I ; X_{2}\right) .
$$

Since the sequence $v^{n}$ is bounded in the space $W^{1, p, 1}\left(0, T ; W_{0, \operatorname{div}}^{1, p}(\Omega), V_{s}^{*}\right)$ and we have the embedding $W^{1, p, 1}\left(0, T ; W_{0, \operatorname{div}}^{1, p}(\Omega), V_{s}^{*}\right) \hookrightarrow \hookrightarrow L^{p}\left(0, T ; L_{\operatorname{div}}^{2}(\Omega)\right)$, hence

$$
v^{n} \rightarrow v \quad \text { strongly in } L^{p}\left(0, T ; L_{\mathrm{div}}^{2}(\Omega)\right) .
$$

This allows us to conclude that for a fixed $i \in \mathbb{N}$ we have

$$
\lim _{n \rightarrow \infty} \int_{0}^{T} \int_{\Omega}\left(v^{n} \cdot \nabla\right) v^{n} \cdot \omega_{i} d x d t=\int_{0}^{T} \int_{\Omega}(v \cdot \nabla) v \cdot \omega_{i} d x d t
$$

Next we will concentrate on the nonlinear term $S\left(x, D v^{n}\right)$. Using Theorem A.2 we conclude that $\chi$ specified in (2.18) is equal to

$$
\chi=\left\langle\nu_{t, x}, S\right\rangle
$$

where $\nu_{t, x}$ is a Young measure generated by the sequence $\nabla v^{n}$. Since $\nabla v^{n}$ is bounded in $L^{p}(Q)$ then the 'tightness condition' is satisfied. Next, using A.2 and A.3, we conclude

$$
\lim _{n \rightarrow \infty} \int_{Q} S\left(x, D v^{n}\right) \cdot D \varphi d x d t=\int_{Q} \int_{\mathbb{R}^{d \times d}} S\left(x, \frac{\xi+\xi^{T}}{2}\right) d \nu_{t, x}(\xi) \cdot D \varphi d x d t
$$

for all $\varphi \in \mathcal{D}((-\infty, T) ; \mathcal{V})$. Choosing $g=i d$ in A.2 we see that

$$
\nabla v^{n} \rightarrow \overline{\nabla v} \text { in } L^{1}(Q)
$$

where

$$
\overline{\nabla v}=\int_{\mathbb{R}^{d \times d}} \xi d \nu_{t, x}(\xi) .
$$

Combining the above equality with

$$
\nabla v^{n} \rightarrow \nabla v \quad \text { weakly in } \quad L^{p}(Q) .
$$


we get (2.10). This proves the first part of the theorem. To prove $(2.11)$ we integrate (2.14) over $(0, \tau)$ with $\tau \in(0, T)$. This gives

$$
\frac{1}{2}\left\|v^{n}(\tau)\right\|_{2}^{2}+\int_{Q_{\tau}} S\left(x, D v^{n}\right) \cdot D v^{n} d x d t=\frac{1}{2}\left\|v^{n}(0)\right\|_{2}^{2}+\int_{0}^{\tau}\left\langle f, v^{n}\right\rangle d t
$$

As $v^{n}(\tau) \rightarrow v(\tau)$ strongly in $L^{2}(\Omega)$ for a.a. $\tau \in I$, and $v_{0}^{n} \rightarrow v_{0}$ strongly in $L^{2}(\Omega)$, letting $n \rightarrow \infty$ in $(2.23)$ we obtain

$$
\frac{1}{2}\|v(\tau)\|_{2}^{2}+\liminf _{n \rightarrow \infty} \int_{Q_{\tau}} S\left(x, D v^{n}\right) \cdot D v^{n} d x d t=\frac{1}{2}\|v(0)\|_{2}^{2}+\int_{0}^{\tau}\langle f, v\rangle d t
$$

for a.a. $\tau \in(0, T)$. Next, since $S\left(x, D v^{n}\right) \cdot D v^{n}$ is nonnegative, we can apply Lemma A.3 to conclude that

$$
\liminf _{n \rightarrow \infty} \int_{Q_{\tau}} S\left(x, D v^{n}\right) \cdot D v^{n} d x d t \geq \int_{Q_{\tau}} \int_{\mathbb{R}^{d \times d}} S\left(x, \frac{\xi+\xi^{T}}{2}\right) \cdot\left(\frac{\xi+\xi^{T}}{2}\right) d \nu_{t, x}(\xi) d x d t .
$$

This together with (2.24) implies (2.11) for almost all $\tau \in(0, T)$.

3. Weak solutions. The existence of weak solutions is not the main issue of the present paper. We provide a short overview of the methods and results concerning existence to ensure that the considerations in the next section are meaningful. For the details of these results we refer to $[4,5]$. Let us first define the weak solutions:

Definition 3.1. We call $v$ a weak solution to (1.1) if

$$
v \in L^{\infty}\left(0, T ; L_{\mathrm{div}}^{2}(\Omega)\right) \cap L^{p}\left(0, T ; W_{0}^{1, p}(\Omega)\right), D v \in L_{M}(Q)
$$

and the following equality is satisfied for all $\varphi \in \mathcal{D}((-\infty, T) ; \mathcal{V})$ :

$$
\int_{Q}\left(-v \cdot \varphi_{t}+(v \cdot \nabla) v \cdot \varphi+S(x, D v) \cdot D \varphi\right) d x d t+\int_{\Omega} v_{0} \cdot \varphi d x=\int_{0}^{T}\langle f, \varphi\rangle d t .
$$

One can show the following result:

TheOREM 3.2. Let $S$ satisfy (S1)-(S4) for $p \geq \frac{3 d+2}{d+2}$ and with $\tilde{M}=M^{*}$, i.e. the complementary function to $M$. Given $f=\operatorname{div} F$ with $F \in L_{M^{*}}(Q)$ and $v_{0} \in L_{\text {div }}^{2}(\Omega)$ there exists a weak solution to (1.1).

Restricting the assumption $(S 4)$ to

$\left(S 4^{\prime}\right) S$ is strictly monotone, i.e. for all $\xi_{1}, \xi_{2} \in \mathbb{R}_{\mathrm{sym}}^{d \times d}, \xi_{1} \neq \xi_{2}$ and a.a. $x \in \Omega$

$$
\left[S\left(x, \xi_{1}\right)-S\left(x, \xi_{2}\right)\right] \cdot\left[\xi_{1}-\xi_{2}\right]>0
$$

one can show that the Young measure $\nu_{t, x}$ generated by the approximate sequence $\left\{\nabla v^{n}\right\}$ is a Dirac measure. This is a direct consequence of the strict monotonicity of the function $S(x, \cdot)$, the weak lower semicontinuity, cf. Lemma A.3 and characterizations $(2.20),(2.22)$. Combining these facts we conclude that

$$
\operatorname{supp}\left\{\nu_{t, x}\right\} \subset\left\{\xi \in \mathbb{R}^{d \times d}: \xi=\nabla v(t, x)\right\}
$$

and since $\nu_{t, x}$ is a probablity measure thus $\nu_{t, x}=\delta_{\nabla v(t, x)}$ a.e. in $Q$. This yields $\chi \stackrel{\text { a.e. }}{=}$ $S(x, D v(t, x))$, which completes the proof of the existence of solutions. This method pro- 
vides further information on solutions like modular convergence of the sequences $\left\{D v^{n}\right\}$ in $L_{M}(Q)$ and $\left\{S\left(x, D v^{n}\right)\right\}$ in $L_{M^{*}}(Q)$.

To provide the proof of Theorem 3.2 under assumptions $(S 1)-(S 4)$ on the stress tensor $S$ we use the generalization of the classical Minty trick, see e.g. [11, 13]. Note that the sequence $\left\{S\left(x, D v^{n}\right)\right\}$ is uniformly bounded in $L_{M^{*}}(Q)$, which in general is not the dual space to $L_{M}(Q)$ and hence the limit passage in appropriate terms fails. However, due to Lemma A.6 and Dunford-Pettis theorem the sequence $\left\{S\left(x, D v^{n}\right)\right\}$ is weakly convergent in $L^{1}(Q)$. Thus to pass to the limit we require that the test function is an element of $L^{\infty}(Q)$. For this reason we introduce the sets

$$
Q_{k}=\{(t, x) \in Q:|D v(t, x)| \leq k \text { a.e. in } Q\}
$$

for any $k>0$ and conclude that

$$
S(x, D v)=\chi \text { a.e. in } Q_{k} .
$$

Since $k$ was arbitrary, $(3.27)$ holds a.e. in $Q$.

4. Uniqueness of solutions. For further consideration we introduce the condition of uniform monotonicity, which is stronger than $\left(S 4^{\prime}\right)$ and consequently $(S 4)$.

$\left(S 4^{\prime \prime}\right)$ There exists $c>0$ such that for all $\xi_{1}, \xi_{2} \in \mathbb{R}_{\mathrm{sym}}^{d \times d}$,

$$
\left(S\left(x, \xi_{1}\right)-S\left(x, \xi_{2}\right)\right) \cdot\left(\xi_{1}-\xi_{2}\right) \geq c\left|\xi_{1}-\xi_{2}\right|^{2} .
$$

Moreover, by $V$ we denote the closure of the space of compactly supported, divergence-free functions $\varphi \in C^{\infty}([0, T) \times \Omega)$ with respect to the modular convergence of the symmetric gradients. Note that in case of $N$-function independent of $x$ if $D v \in L_{M}(Q)$, one can prove $v \in V$, cf. [3]. In case of $x$-dependent $N$-functions we can show an analogous result under the assumption of strict monotonicity of $S$ for the limit of Galerkin approximation.

THEOREM 4.1. Let $S$ satisfy $(S 1)-\left(S 4^{\prime \prime}\right)$ for $p \geq \frac{d+2}{2}$ and with $\tilde{M}=M^{*}$, i.e. the complementary function to $M$. Moreover let $v$ be the weak solution to (1.1) for given $f=\operatorname{div} F$ with $F \in L_{M^{*}}(Q)$ and $v_{0} \in L_{\mathrm{div}}^{2}(\Omega)$. If $v \in C\left(0, T ; L_{\mathrm{div}}^{2}(\Omega)\right) \cap V$, then it is the unique solution to (1.1).

Proof. Let us define a continuous function $\theta_{l}$ as follows:

$$
\theta_{l}(t)=\left\{\begin{array}{cc}
1 & s_{0}+\frac{2}{l}<t<s_{1}-\frac{2}{l} \\
0 & t<s_{0}+\frac{1}{l}, s_{1}-\frac{1}{l}<t \\
\text { linear } & \text { otherwise }
\end{array}\right.
$$

Moreover, let $\varrho^{\varepsilon}$ be a standard mollifier, i.e., $\varrho \in C^{\infty}(\mathbb{R})$, $\varrho$ has a compact support and $\int_{\mathbb{R}} \varrho(\tau) d \tau=1, \varrho(t)=\varrho(-t)$. We define $\varrho^{\varepsilon}(t)=\frac{1}{\varepsilon} \varrho\left(\frac{t}{\varepsilon}\right)$. Now let $u$ and $v$ be two weak solutions to (1.1) with the same initial condition. We define $w=u-v$ and $w_{l}^{\varepsilon}=$ $\theta_{l}\left(\varrho^{\varepsilon} * \varrho^{\varepsilon} * \theta_{l} w\right)$, where $*$ denotes the convolution in the variable $t$ and $\varepsilon<1 / l$. Subtracting the weak formulation for $u$ and $v$ and taking $w_{l}^{\varepsilon}$ as a test function we obtain

$$
\begin{aligned}
-\int_{Q} w \cdot\left(w_{l}^{\varepsilon}\right)_{t} d x d t & +\int_{Q}((u \cdot \nabla) u-(v \cdot \nabla) v) \cdot w_{l}^{\varepsilon} d x d t \\
& +\int_{Q}(S(x, D u)-S(x, D v)) \cdot D w_{l}^{\varepsilon} d x d t=0 .
\end{aligned}
$$


We wish to let $\varepsilon \rightarrow 0$ and $l \rightarrow \infty$. Note that

$$
\begin{aligned}
\int_{Q} w \cdot\left(w_{l}^{\varepsilon}\right)_{t} d x d t & =\int_{Q} w \cdot\left(\theta_{l} \varrho^{\varepsilon} * \varrho^{\varepsilon} * \theta_{l} w\right)_{t} d x d t \\
& =\int_{Q}\left(\theta_{l} w\right) \cdot\left(\varrho^{\varepsilon} * \varrho^{\varepsilon} * \theta_{l} w\right)_{t} d x d t+\int_{Q}\left(\theta_{l}^{\prime} w\right) \cdot\left(\varrho^{\varepsilon} * \varrho^{\varepsilon} * \theta_{l} w\right) d x d t \\
& =\int_{Q}\left(\varrho^{\varepsilon} *\left(\theta_{l} w\right)\right) \cdot\left(\varrho^{\varepsilon} *\left(\theta_{l} w\right)_{t}\right) d x d t+\int_{Q}\left(\theta_{l}^{\prime} w\right) \cdot\left(\varrho^{\varepsilon} * \varrho^{\varepsilon} * \theta_{l} w\right) d x d t .
\end{aligned}
$$

The first integral of the right-hand side is equal to zero. Note that $w \in C\left(0, T ; L_{\mathrm{div}}^{2}(\Omega)\right)$ and hence for all $0<s_{0}<s<T$,

$$
\lim _{l \rightarrow \infty} \lim _{\varepsilon \rightarrow 0} \int_{Q} w \cdot\left(w_{l}^{\varepsilon}\right)_{t} d x d t=\frac{1}{2}\|w(s)\|_{2}^{2}-\frac{1}{2}\left\|w\left(s_{0}\right)\right\|_{2}^{2} .
$$

Now we will consider the trilinear term. Since $p \geq \frac{d+2}{2}$, we have

$$
\begin{aligned}
& \lim _{l \rightarrow \infty} \lim _{\varepsilon \rightarrow 0} \int_{Q}((u \cdot \nabla) u-(v \cdot \nabla) v) \cdot w_{l}^{\varepsilon} d x d t=\lim _{l \rightarrow \infty} \lim _{\varepsilon \rightarrow 0} \int_{Q}((u-v) \cdot \nabla) u \cdot w_{l}^{\varepsilon} d x d t \\
& +\lim _{l \rightarrow \infty} \lim _{\varepsilon \rightarrow 0} \int_{Q}(v \cdot \nabla)(u-v) \cdot w_{l}^{\varepsilon} d x d t=\int_{s_{0}}^{s} \int_{\Omega}((u-v) \cdot \nabla) u \cdot w d x d t .
\end{aligned}
$$

Consider now the nonlinear term

$$
\begin{aligned}
& \int_{Q}(S(x, D u)-S(x, D v)) \cdot\left(\theta_{l}\left(\varrho^{\varepsilon} * \varrho^{\varepsilon} * \theta_{l} D w\right)\right) d x d t= \\
& \int_{Q}\left(\varrho^{\varepsilon} * \theta_{l}(S(x, D u)-S(x, D v))\right) \cdot\left(\varrho^{\varepsilon} * \theta_{l} D w\right) d x d t .
\end{aligned}
$$

Both of the sequences $\left\{\varrho^{\varepsilon} * \theta_{l}(S(x, D u)-S(x, D v))\right\}$ and $\left\{\varrho^{\varepsilon} * \theta_{l} D w\right\}$ converge in measure in $Q$ due to Proposition A.9. Moreover, since $M$ and $M^{*}$ are convex nonnegative functions, the weak lower semicontinuity and estimate (2.17) show that the integrals

$$
\int_{Q} M(x, D w) d x d t \quad \text { and } \quad \int_{Q} M^{*}(x,(S(x, D u)-S(x, D v))) d x d t
$$

are finite. Hence Proposition A.10 implies that $\left\{\varrho^{\varepsilon} * \theta_{l}(S(x, D u)-S(x, D v))\right\}$ and also $\left\{\varrho^{\varepsilon} * \theta_{l} D w\right\}$ are uniformly bounded and hence according to Proposition A.6 we have

$$
\begin{gathered}
\varrho^{\varepsilon} * \theta_{l} D w \stackrel{M}{\longrightarrow} \theta_{l} D w \quad \text { in } L_{M}(Q), \\
\varrho^{\varepsilon} * \theta_{l}(S(x, D u)-S(x, D v)) \stackrel{M^{*}}{\longrightarrow} \theta_{l}(S(x, D u)-S(x, D v)) \quad \text { in } L_{M^{*}}(Q),
\end{gathered}
$$

as $\varepsilon \rightarrow 0$. Applying Proposition A.8 we conclude

$$
\begin{gathered}
\lim _{l \rightarrow \infty} \lim _{\varepsilon \rightarrow 0} \int_{Q}\left(\varrho^{\varepsilon} * \theta_{l}(S(x, D u)-S(x, D v))\right) \cdot\left(\varrho^{\varepsilon} * \theta_{l} D w\right) d x d t \\
=\int_{s_{0}}^{s} \int_{\Omega}(S(x, D u)-S(x, D v)) \cdot D w d x d t \geq 0 .
\end{gathered}
$$


Combining (4.31), (4.34) and (4.32) we obtain

$$
\begin{aligned}
\frac{1}{2}\|w(s)\|_{2}^{2}+ & \int_{s_{0}}^{s} \int_{\Omega}(S(x, D u)-S(x, D v)) \cdot D w d x d t \\
& =\frac{1}{2}\left\|w\left(s_{0}\right)\right\|_{2}^{2}-\int_{s_{0}}^{s} \int_{\Omega}(w \cdot \nabla) u \cdot w d x d t
\end{aligned}
$$

for almost all $0<s_{0}<s<T$. Since $w \in C\left([0, T) ; L_{\text {div }}^{2}(\Omega)\right)$, then we can choose a sequence $s_{0}^{n} \rightarrow 0$ such that

$$
w\left(s_{0}^{n}\right) \rightarrow w(0) \quad \text { in } L_{\mathrm{div}}^{2}(\Omega)
$$

and

$$
\lim _{n \rightarrow \infty}\left\|w\left(s_{0}^{n}\right)\right\|_{2}=\|w(0)\|_{2} .
$$

Choosing $s_{0}=s_{0}^{n}$ in (4.35) and letting $n \rightarrow \infty$ we obtain

$$
\begin{aligned}
\frac{1}{2}\|w(s)\|_{2}^{2}+ & \int_{0}^{s} \int_{\Omega}(S(x, D u)-S(x, D v)) \cdot D w d x d t \\
& =\frac{1}{2}\|w(0)\|_{2}^{2}-\int_{0}^{s} \int_{\Omega}(w \cdot \nabla) u \cdot w d x d t .
\end{aligned}
$$

By Hölder's inequality

$$
\int_{0}^{s} \int_{\Omega}(w \cdot \nabla) u \cdot w d x d t \leq \int_{0}^{s} \int_{\Omega}|w|^{2}|\nabla u| d x d t \leq \int_{0}^{s}\|\nabla u\|_{p}\|w\|_{\frac{2 p}{p-1}}^{2} d t .
$$

Combining (4.37), (4.38), condition (4.28) and Korn's inequality we obtain

$$
\frac{1}{2}\|w(s)\|_{2}^{2}+K_{1} \int_{0}^{s}\|\nabla w\|_{2}^{2} d t \leq \int_{0}^{s}\|\nabla u\|_{p}\|w\|_{\frac{2 p}{p-1}}^{2} d t .
$$

By the interpolation inequality (4.40) we get

$$
\begin{aligned}
\frac{1}{2}\|w(s)\|_{2}^{2}+K_{1} \int_{0}^{s}\|\nabla w\|_{2}^{2} d t & \leq \int_{0}^{s}\|\nabla u\|_{p}\|w\|_{\frac{2 p-d}{p}}^{2} d t \\
& \stackrel{\leq}{\leq} K_{1} \int_{0}^{s}\|\nabla w\|_{2}^{2} d t+K_{2} \int_{0}^{s}\|\nabla u\|_{2}^{2} d t
\end{aligned}
$$

From Gronwall's Lemma we obtain

$$
\|w(s)\|_{2}=0 \quad \text { for a.a. } s \in[0, T) .
$$

Thus $u(s)=v(s)$ almost everywhere in $[0, T)$.

Lemma 4.2. Let $v \in W_{0}^{1,2}(\Omega)$ and $q \in\left[2, \frac{2 d}{d-2}\right]$ for $d \geq 3$ and $q \in(2,+\infty)$ if $d=2$. Then there exists $c>0$ such that

$$
\|v\|_{q} \leq c\|v\|_{2}^{\alpha}\|\nabla v\|_{2}^{1-\alpha}
$$

with $\alpha=\frac{2 d-q(d-2)}{2 q}$. 


\section{A. Appendix}

A.1. Young measures. The proofs of the following facts can be found in $[9$, Corollaries 3.2-3.4], $[1,8]$. In the following $C_{0}\left(\mathbb{R}^{d}\right)$ denotes the closure of the space of continuous functions on $\mathbb{R}^{d}$ with compact support with respect to the $\|\cdot\|_{\infty}$-norm. Its dual space can be identified with $\mathcal{M}\left(\mathbb{R}^{d}\right)$, the space of signed Radon measures with finite mass. The related duality pairing is given by

$$
\langle\mu, f\rangle=\int_{\mathbb{R}^{d}} f(\lambda) d \mu(\lambda) .
$$

Definition A.1. A map $\mu: \Omega \rightarrow \mathcal{M}\left(\mathbb{R}^{d}\right)$ is called weakly* measurable if the functions $x \rightarrow\langle\mu(x), f\rangle$ are measurable for all $f \in C_{0}\left(\mathbb{R}^{d}\right)$.

Theorem A.2 (Fundamental theorem on Young measures). Let $\Omega \subset \mathbb{R}^{d}$ be a measurable set of finite measure and let $z^{j}: \Omega \rightarrow \mathbb{R}^{d}$ be a sequence of measurable functions. Then there exists a subsequence $z^{j_{k}}$ and a weakly* measurable map $\nu: \Omega \rightarrow \mathcal{M}\left(\mathbb{R}^{d}\right)$ such that:

(i) $\nu_{x} \geq 0, \quad\left\|\nu_{x}\right\|_{\mathcal{M}\left(\mathbb{R}^{d}\right)}=\int_{\mathbb{R}^{d}} d \nu_{x} \leq 1 \quad$ for a.a. $x \in \Omega$.

(ii) For all $g \in C_{0}\left(\mathbb{R}^{d}\right)$

$$
\left(z^{j_{k}}\right) \stackrel{*}{\rightarrow} \bar{g} \text { in } L^{\infty}(\Omega) \text { where } \bar{g}(x)=\left\langle\nu_{x}, g\right\rangle .
$$

(iii) Additionally $\left\|\nu_{x}\right\|_{\mathcal{M}\left(\mathbb{R}^{d}\right)}=1$ for a.a. $x \in \Omega$ if and only if the 'tightness condition' is satisfied, i.e.

$$
\lim _{M \rightarrow \infty} \sup _{k}\left|\left\{\left|z^{j_{k}}\right| \geq M\right\}\right|=0 .
$$

(iv) If the tightness condition is satisfied and moreover if $A \subset \Omega$ is measurable, $g \in$ $C\left(\mathbb{R}^{d}\right)$ and $g\left(z^{j_{k}}\right)$ is relatively weakly compact in $L^{1}(A)$, then

$$
g\left(z^{j_{k}}\right) \rightarrow \bar{g} \quad \text { in } L^{1}(A), \quad \bar{g}(x)=\left\langle\nu_{x}, g\right\rangle .
$$

REMARK. The map $\nu: \Omega \rightarrow \mathcal{M}\left(\mathbb{R}^{d}\right)$ is called the Young measure generated by the sequence $z^{j_{k}}$. Every (weakly* measurable map) $\nu: \Omega \rightarrow \mathcal{M}\left(\mathbb{R}^{d}\right)$ that satisfies $(i)$ is generated by some sequence $z^{k}$.

REMARK. If $\int_{\Omega}\left|z^{j}\right|^{s} \leq k$ for some $s>0$ and all $j \in \mathbb{N}$, then the tightness condition is satisfied.

Lemma A.3. Suppose that the sequence of measurable functions $z^{j}: \Omega \rightarrow \mathbb{R}^{d}$ generates the Young measure $\nu: \Omega \rightarrow \mathcal{M}\left(\mathbb{R}^{d}\right)$. Let $F: \Omega \times \mathbb{R}^{d} \rightarrow \mathbb{R}$ be a Carathéodory function. Let us also assume that the negative part $F^{-}\left(x, z^{j}(x)\right)$ is weakly relatively compact in $L^{1}(\Omega)$. Then

$$
\liminf _{j \rightarrow \infty} \int_{\Omega} F\left(x, z^{j}(x)\right) d x \geq \int_{\Omega} \int_{\mathbb{R}^{d}} F(x, \lambda) d \nu_{x}(\lambda) d x .
$$

If, in addition, the sequence of functions $x \mapsto|F|\left(x, z^{j}(x)\right)$ is weakly relatively compact in $L^{1}(\Omega)$ then

$$
F\left(\cdot, z^{j}(\cdot)\right) \rightarrow \int_{\mathbb{R}^{d}} F(x, \lambda) d \nu_{x}(\lambda) \quad \text { in } \quad L^{1}(\Omega)
$$


A.2. Generalized Orlicz spaces. For completeness we recall some general facts on Orlicz spaces, see e.g. [10] and technical facts proved in [4].

Proposition A.4 (Fenchel-Young Inequality). Let $M$ be an $N$-function and $M^{*}$ the complementary function to $M$. Then

$$
|\xi \cdot \eta| \leq M(x, \xi)+M^{*}(x, \eta)
$$

for all $\xi, \eta \in \mathbb{R}_{\mathrm{sym}}^{d \times d}$ and a.a. $x \in \Omega$.

Proposition A.5. If $M$ satisfies the $\Delta_{2}$-condition (1.6), then $\mathcal{L}_{M}(Q)$ is a vector space.

Next, we recall an analogue to Vitali's lemma, but for the modular convergence instead of the strong convergence in $L^{p}$.

Lemma A.6. Let $z^{j}: Q \rightarrow \mathbb{R}^{d}$ be a measurable sequence. Then $z^{j} \stackrel{M}{\longrightarrow} z$ in $L_{M}(Q)$ modularly if and only if $z^{j} \rightarrow z$ in measure and there exist some $\lambda>0$ such that the sequence $\left\{M\left(\cdot, \lambda z^{j}\right)\right\}$ is uniformly integrable, i.e.,

$$
\lim _{R \rightarrow \infty}\left(\sup _{j \in \mathbb{N}} \int_{\left\{(t, x):\left|M\left(x, \lambda z^{j}\right)\right| \geq R\right\}} M\left(x, \lambda z^{j}\right) d x d t\right)=0 .
$$

The following technical facts were used in the paper:

LEmmA A.7. Let $M$ be an $N$-function and for all $j \in \mathbb{N}$ let $\int_{Q} M\left(x, z^{j}\right) d x d t \leq c$. Then the sequence $\left\{z^{j}\right\}$ is uniformly integrable.

Proposition A.8. Let $M$ be an $N$-function and $M^{*}$ its complementary function. Suppose that the sequences $\psi^{j}: Q \rightarrow \mathbb{R}^{d}$ and $\phi^{j}: Q \rightarrow \mathbb{R}^{d}$ are uniformly bounded in $L_{M}(Q)$ and $L_{M^{*}}(Q)$ respectively. Moreover $\psi^{j} \stackrel{M}{\longrightarrow} \psi$ modularly in $L_{M}(Q)$ and $\phi^{j} \stackrel{M^{*}}{\longrightarrow} \phi \operatorname{modu-}$ larly in $L_{M^{*}}(Q)$. Then $\psi^{j} \cdot \phi^{j} \rightarrow \psi \cdot \phi$ strongly in $L^{1}(Q)$.

Proposition A.9. Let $\varrho^{j}$ be a standard mollifier, i.e., $\varrho \in C^{\infty}(\mathbb{R}), \varrho$ has a compact support and $\int_{\mathbb{R}} \varrho(\tau) d \tau=1, \varrho(t)=\varrho(-t)$. We define $\varrho^{j}(t)=j \varrho(j t)$. Moreover let $*$ denote convolution in the variable $t$. Then for any function $\psi: Q \rightarrow \mathbb{R}^{d}$ such that $\psi \in L^{1}(Q)$,

$$
\left(\varrho^{j} * \psi\right)(t, x) \rightarrow \psi(t, x) \quad \text { in measure. }
$$

Proposition A.10. Let $\varrho^{j}$ be defined as in Proposition A.9. Given an $N$-function $M$ and a function $\psi: Q \rightarrow \mathbb{R}^{d}$ such that $\psi \in \mathcal{L}_{M}(Q)$. Then the sequence $\left\{M\left(x, \varrho^{j} * \psi\right)\right\}$ is uniformly integrable.

Acknowledgements. P.G. and A.S.-G. were supported by the Grant of Ministry of Science and Higher Education, No. N201 033 32/2269. P.G. appreciates the return fellowship of Alexander von Humboldt Foundation.

\section{References}

[1] J. M. Ball, A version of the fundamental theorem for Young measures, in: PDEs and Continuum Models of Phase Transitions (Nice, 1988), Lecture Notes in Phys. 344, Springer, Berlin, 1989, 207-215. 
[2] M. Bulíček, F. Ettwein, P. Kaplický, and D. Pražák, The regularity of non-Newtonian flows, submitted, 2008.

[3] J.-P. Gossez, Some approximation properties in Orlicz-Sobolev spaces, Studia Math. 74 (1982), 17-24.

[4] P. Gwiazda and A. Świerczewska-Gwiazda, On non-Newtonian fluids with a property of rapid thickening under different stimulus, Math. Models Methods Appl. Sci. 18 (2008), 1073-1092.

[5] P. Gwiazda, A. Świerczewska-Gwiazda, and A. Wróblewska, Monotonicity methods in generalized Orlicz spaces for a class of non-newtonian fluids, Math. Methods Appl. Sci., (2008).

[6] O. Ladyzhenskaya, The Mathematical Theory of Viscous Incompressible Flows, Gordon and Breach, New York, 1969.

[7] J.-L. Lions, Quelques méthodes de résolution des problèmes aux limites non linéaires, Dunod, 1969.

[8] J. Málek, J. Nečas, M. Rokyta, and M. Růžička, Weak and Measure-Valued Solutions to Evolutionary PDEs, Chapman \& Hall, London, 1996.

[9] S. Müller, Variational models for microstructure and phase transitions, in: Calculus of Variations and Geometric Evolution Problems (Cetraro, 1996), Lecture Notes in Math. 1713, Springer, Berlin, 1999, 85-210.

[10] J. Musielak, Orlicz Spaces and Modular Spaces, Lecture Notes in Math. 1034, Springer, Berlin, 1983.

[11] V. Mustonen and M. Tienari, On monotone-like mappings in Orlicz-Sobolev spaces, Math. Bohem. 124 (1999), 255-271.

[12] T. Roubíček, Nonlinear Partial Differential Equations with Applications, International Series of Numerical Mathematics 153, Birkhäuser Verlag, Basel, 2005.

[13] A. Wróblewska, Existence of solutions to non-Newtonian steady flows in generalized Orlicz spaces - monotonicity methods, submitted, 2008. 